\title{
Heavy quark results from STAR
}

\author{
Xin Dong ${ }^{\mathrm{a}}$ \\ for the STAR Collaboration \\ Lawrence Berkeley National Lab, Berkeley, CA 94720, USA
}

Received: 12 September 2008 / Revised: 3 December 2008 / Published online: 21 February 2009

(C) Springer-Verlag / Società Italiana di Fisica 2009

\begin{abstract}
I report the most recent measurements on open heavy flavor production at RHIC on behalf of the STAR collaboration. The total charm production cross section in midrapidity at RHIC energy is found to approximately scale by number of binary collisions in $d+\mathrm{Au}, \mathrm{Cu}+\mathrm{Cu}$ and $\mathrm{Au}+\mathrm{Au}$ collisions. The nuclear modification factor of nonphotonic electrons is strongly suppressed in central $\mathrm{Au}+\mathrm{Au}$ collisions, suggesting substantial heavy quark energy loss at RHIC. The bottom decay contribution to non-photonic electrons was studied via the $e-h$ and $e-D^{0}$ azimuthal angular correlations. The bottom contribution is found to be important at $p_{T}>5 \mathrm{GeV} / c$, and is consistent with the FONLL calculation within uncertainties. Charm production through gluon jet splitting was measured by studying the $D^{* \pm}$ contents in the fully reconstructed jets in $p+p$ collisions. This rate is consistent with $\mathrm{pQCD}$ evaluation of gluon splitting into a pair of charm quarks and subsequent hadronization.
\end{abstract}

PACS 25.75.Dw $\cdot$ 13.20.Fc $\cdot 13.25 . \mathrm{Ft} \cdot 24.85 .+\mathrm{p}$

\section{Introduction}

Heavy quark production is believed to be a powerful test for pQCD calculations in elementary collisions. The theoretical predictions for heavy quark production cross section at high energies are extensively reported in [1, 2]. In heavy ion collisions, charm yields are expected to scale by $N_{\text {bin }}$ since most charm quark pairs are created in the initial hard processes. Due to their large mass, charm and bottom quark masses are not likely to be modified in the QCD medium. Hence heavy quark collectivity is a better probe of the early stage than light quarks, and thus indicates early thermalization of the lighter quarks. Measurements on open charm production also provide an important reference for charmonium production study, which is of great significance in searching

a e-mail: xdong@lbl.gov and quantifying the QGP. In this article, I will report the recent experimental results on open heavy quark production at RHIC collisions from the STAR experiment.

\section{Recent open heavy quark measurements from STAR}

Table 2.1 lists the measurements made so far at STAR on the charm production spectra via various methods in different collision systems (shown as checkmarks). There are three independent methods: low $p_{T} D^{0}$ reconstruction through the hadronic decay channel, non-photonic electrons, and heavy flavor decay muons. In the non-photonic electron approach, there are several independent analyses which involve three different subsystems: the Time Projection Chamber (TPC), the Time-Of-Flight (TOF) detector and the Electromagnetic Calorimeter (EMC) [3-5]. So far, all available results from these measurements are consistent with each other in each overlap area.

The large coverage of the TPC would allow us to reconstruct charm mesons (like $D^{0}$ ) through its hadronic decay channels benefiting from the large statistics to overcome large combinatorial background. Figure 2.1 shows the unlike charge-sign $K \pi$ invariant mass distributions after combinatorial background subtraction for $D^{0}+\overline{D^{0}}$ candidates at low $p_{T}\left(p_{T} \lesssim 3 \mathrm{GeV} / c\right)$ in $d+\mathrm{Au}, \mathrm{Au}+\mathrm{Au}$ and $\mathrm{Cu}+$ $\mathrm{Cu}$ minimum bias collisions $[3,4,6]$. The observed $D^{0}$ signal significances are $\sim 4-6 \sigma$ in these systems, and the $S / B$ ratios are around 1/3000 $(\mathrm{Au}+\mathrm{Au})-1 / 600(d+\mathrm{Au})$.

The semi-leptonic decay channel is an alternative approach to measure heavy quark production to reach higher $p_{T}$ and different kinematic regions. In STAR, the TOF and EMC detectors can help improve the $e / h$ ratio by several orders of magnitude. One can refer to the publications [3-5] for technical details. Background electrons, which come from the inner detector conversions and light hadron electromagnetic decays (photonic electrons), were removed by the cut on small invariant mass of this electron and a partner 
Table 2.1 Measurements made at STAR on the charm spectra via various methods in different collision systems

\begin{tabular}{|c|c|c|c|c|c|}
\hline \multirow[b]{2}{*}{ Sub-systems } & \multirow{2}{*}{$\begin{array}{l}\text { D reconstruction } \\
\text { TPC }\end{array}$} & \multicolumn{3}{|c|}{ non-photonic electrons } & \multirow{2}{*}{$\begin{array}{l}\text { low } p_{T} \text { muons } \\
\text { TPC+TOF }\end{array}$} \\
\hline & & $\mathrm{TPC}+\mathrm{TOF}$ & TPC & $\mathrm{TPC}+\mathrm{EMC}$ & \\
\hline$p_{T}$ coverage $(\mathrm{GeV} / c)$ & $\sim 0.1-3$ & $\sim 0.8-5$ & $\sim 2-4$ & $\sim 2-10$ & $\sim 0.17-0.25$ \\
\hline$p+p$ & - & $\checkmark$ & $\checkmark$ & $\checkmark$ & - \\
\hline$d+\mathrm{Au}$ & $\checkmark$ & $\checkmark$ & $\checkmark$ & $\checkmark$ & - \\
\hline $\mathrm{Cu}+\mathrm{Cu}$ & $\checkmark$ & - & - & $\checkmark$ & - \\
\hline $\mathrm{Au}+\mathrm{Au}$ & $\checkmark$ & $\checkmark$ & - & $\checkmark$ & $\checkmark$ \\
\hline
\end{tabular}

Fig. 2.1 Invariant mass distributions for $D^{0}+\overline{D^{0}}$ candidates from $K \pi$ decay channel at low $p_{T}$ in $d+\mathrm{Au}$ (upper left), $\mathrm{Au}+\mathrm{Au}$ (upper right) and $\mathrm{Cu}+\mathrm{Cu}$ (lower) minimum bias collisions in $\sqrt{s_{N N}}=200 \mathrm{GeV}$
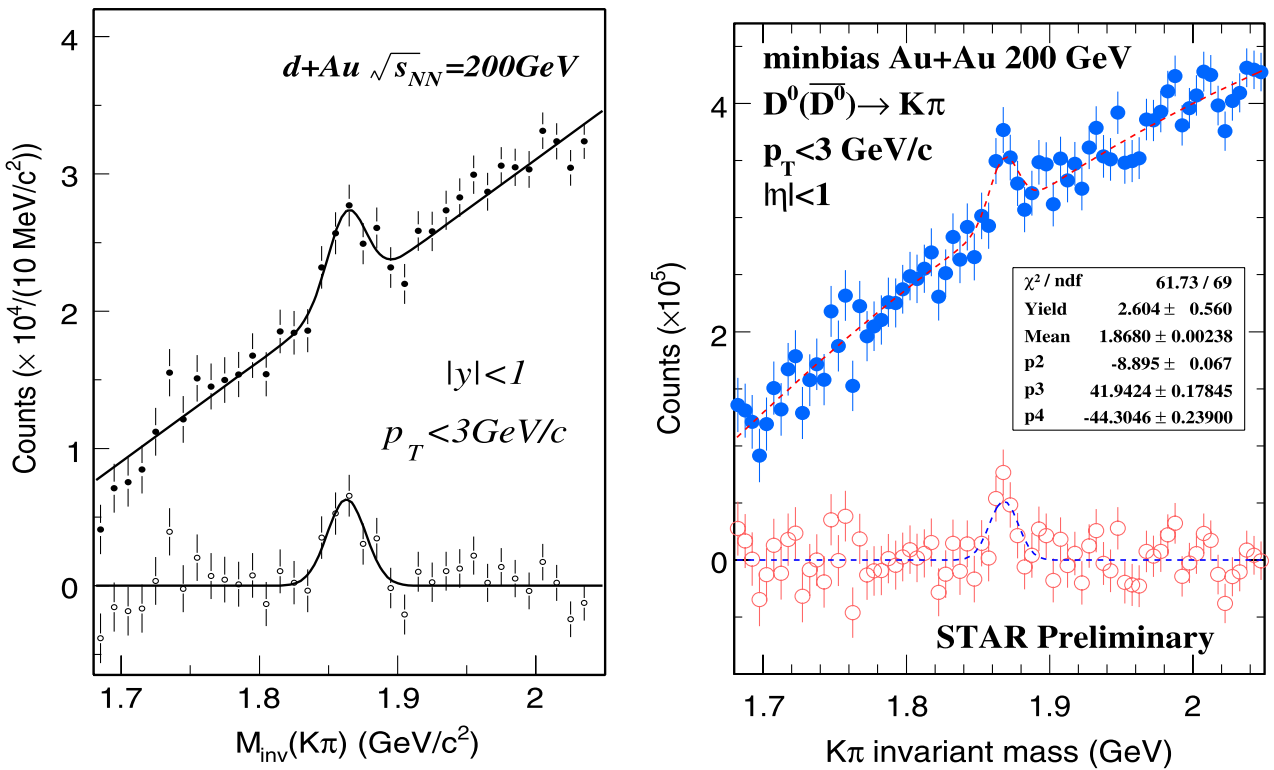

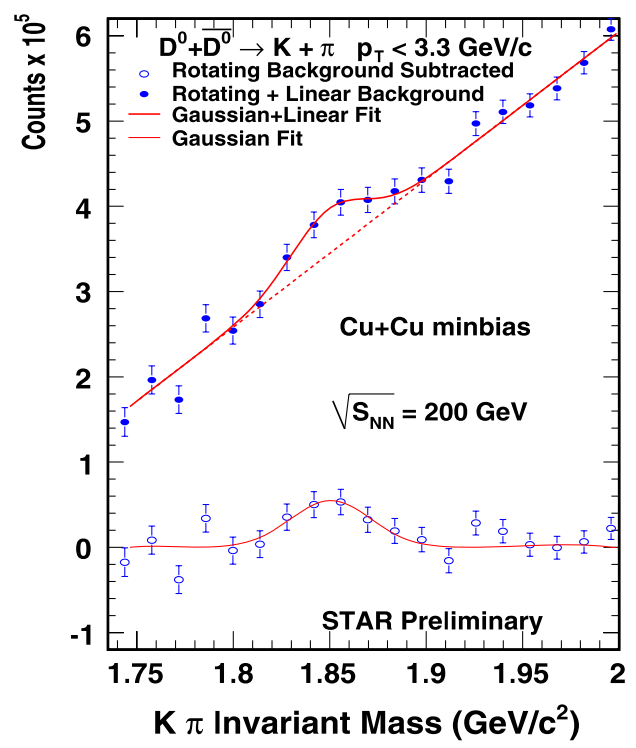

positron. The partner finding efficiency was estimated from the Monte Carlo simulations, which is $\sim 50 \%$ at low $p_{T}$ and increases to $\sim 80 \%$ at high $p_{T}$. Background subtracted electron (non-photonic electron) spectra from TPC+TOF and
TPC +EMC combinations in various collision systems are shown in Fig. 2.2.

By combining the TOF and TPC, one can identify muons at $0.17<p_{T}<0.25 \mathrm{GeV} / c$ in $\mathrm{Au}+\mathrm{Au}$ collisions [4]. Low 

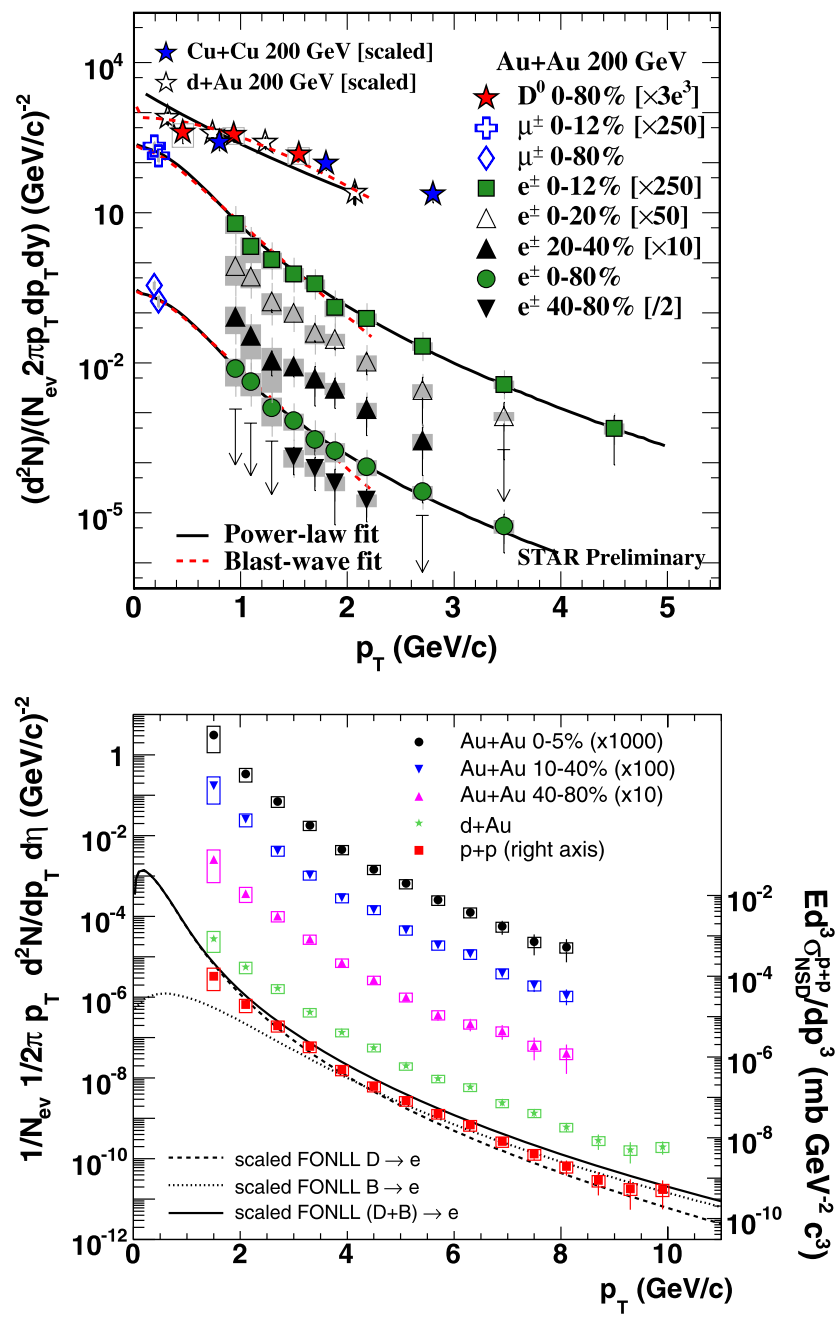

Fig. 2.2 Upper: $D^{0}$, non-photonic electron and heavy flavor decay $\mu$ transverse momentum spectra from $\mathrm{A}+\mathrm{A}$ collisions at low $p_{T}$. Lower: Non-photonic electron transverse momentum spectra from various systems at high $p_{T}$

$p_{T}$ muons are interesting because the yield of those muons is sensitive to the total charm cross section, and doesn't change much with the charm spectrum shape [7]. Therefore, the muon measurement will have a significant impact on the charm total cross section measurement. By statistical subtraction of the muons from light hadron weak decays, one obtains the muon yield which is expected from the heavy flavor decays. The data points in $\mathrm{Au}+\mathrm{Au}$ collisions are shown in Fig. 2.2 upper plot.

To take advantage of all available measurements, we perform combined fits to all available data points on $D^{0}, \mu$, and non-photonic electrons in each collision system [3, 4] (fit to $D^{0}$ data points only in $\mathrm{Cu}+\mathrm{Cu}$ collisions). The results from $d+\mathrm{Au}, \mathrm{Cu}+\mathrm{Cu}, \mathrm{Au}+\mathrm{Au}$ minimum bias, and $\mathrm{Au}+\mathrm{Au}$ central collisions are shown in Fig. 2.3. The results show that the total charm cross section scales with number of binary collisions from $d+\mathrm{Au}$ collisions to $\mathrm{Au}+\mathrm{Au}$ central collisions with the current experimental uncertainty. A pre-

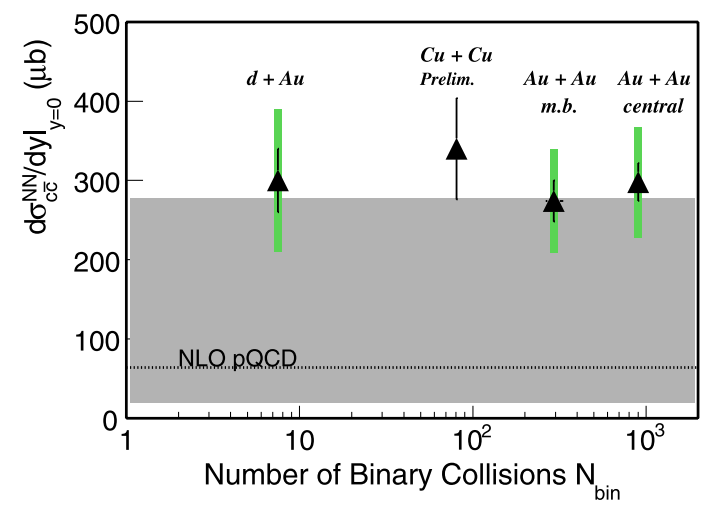

Fig. 2.3 The extract charm cross sections at mid-rapidity from various collision system compared with the NLO pQCD calculations. Statistical and systematical errors are depicted by the black lines and green bars, respectively

cision measurement on the total charm cross section in various collision systems is important because the total charm cross section is an input in the calculation of charmonium production yields through coalescence process, and the charmonium suppression or enhancement could be a QGP signature in heavy ion collisions. The cross section results are also compared with the recent NLO pQCD calculation [1,2] on the total charm cross section on that figure. The finding from the NLO calculations is that the theoretical uncertainties on the charm cross section are quite large. Within the usual variations of the calculation, including charm quark mass, factorization and renormalization scales, the total calculated cross section and the experimentally measured one are consistent with each other.

The particle transverse momentum spectrum in the low $p_{T}$ region can often be fit to the Blast-Wave (BW) model and depict the flow and freeze-out characteristics. To study whether charm hadrons have similar radial flow to light hadrons, the data points of the nuclear modification factors ( $R_{A A}, d+$ Au data points used as the baseline) for $\mu$ and non-photonic electrons, shown in Fig. 2.4 upper plot, are compared with those curves for the expected $R_{A A}$ from a BW model using the freeze-out parameters for light hadrons (BW3 in Fig. 2.4 upper plot) and multi-strange hadrons (BW2) [4]. These two curves miss the data points by a large distance, while the best $\mathrm{BW}$ fit to the $D^{0}, \mu$ and non-photonic electron data points show significant differences from both these curves for $p_{T}>1 \mathrm{GeV} / c$, which suggests that the charm hadron freeze-out and flow are different from light hadrons. The $\chi^{2}$ scan in a 2-dimensional $T_{f o}$ (freeze-out temperature), $\left\langle\beta_{t}\right\rangle$ (mean transverse velocity) space shows little sensitivity to the freeze-out temperature, but disfavors large radial flow. These findings, together with the observation of large charm elliptic flow, are consistent with the recent prediction from hydrodynamics: elliptic flow is built up at partonic stage, and radial flow dominantly 

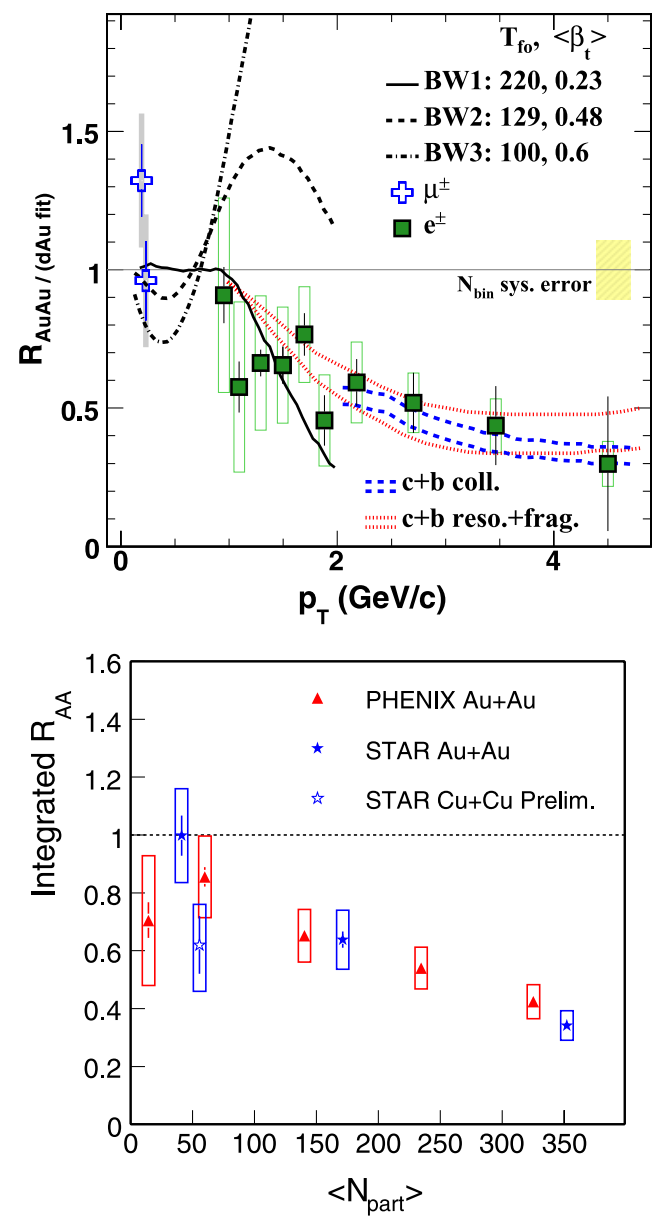

Fig. 2.4 Upper: Nuclear modification factor $\left(R_{\mathrm{AuAu} / d \mathrm{Au}}\right)$ for $\mu$ and non-photonic electrons in $0-12 \% \mathrm{Au}+\mathrm{Au}$ collisions. The data points are compared with the calculations from the BW models with several different sets of freeze-out parameters at low $p_{T}$, and energy loss models at high $p_{T}$. Lower: Integrated $R_{A A}\left(p_{T}>3 \mathrm{GeV} / c\right)$ of non-photonic electrons from different centralities in $\mathrm{Au}+\mathrm{Au}$ collisions and $\mathrm{Cu}+\mathrm{Cu}$ minimum bias collision

comes from hadronic scattering at a later stage where charm hadron may have already decoupled from the system [4].

Figure 2.4 upper plot also shows at high $p_{T}$, the nonphotonic electron $R_{A A}$ is strongly suppressed, indicating the heavy quark may also lose significant amount of energy when traversing through the medium. The results from TPC+TOF and TPC+EMC measurements are consistent $[4,5]$. Recent non-photonic electron measurements in $\mathrm{Cu}+\mathrm{Cu}$ collisions show that significant suppression also exists in $\mathrm{Cu}+\mathrm{Cu}$ system. Figure 2.4 lower plot shows the integrated $R_{A A}\left(p_{T}>3 \mathrm{GeV} / c\right)$ of non-photonic electrons vs. number of participants in various systems. The preliminary measurement in $\mathrm{Cu}+\mathrm{Cu}$ shows the $R_{A A}$ suppression of non-photonic electron follows the same $N_{\text {part }}$ dependence as the $\mathrm{Au}+\mathrm{Au}$ data. The discovery of the strong suppression in non-photonic electron $R_{A A}$ challenges theorists to revisit the energy loss mechanisms.
On the experimental side of the non-photonic electron research, the main hindrance is the difficulty to distinguish the contributions of charm and bottom separately. On the theory side, the FONLL calculation of the $p_{T}$ spectrum predicts the crossing point between electrons coming from charm and those from bottom decays can happen anywhere between $\sim 3-10 \mathrm{GeV} / c[1,2]$. One must therefore rely on additional measurements to pin this down. STAR tried a couple of approaches to extract the bottom contribution via non-photonic electron triggered correlation analyses.

The first one is by measuring the azimuthal angular correlations between non-photonic electrons and charged hadrons in $p+p$ collisions $[8,9]$. Due to the larger mass of bottom hadrons, the near-side correlation function width is expected to be larger in the semi-leptonic decay of a $B$ meson compared to a $D$ meson. We obtained the distributions for charm meson decay and bottom meson decay based on Monte Carlo simulation (PYTHIA), and then simultaneously fit the experimental correlation function with these two distributions and obtain the relative contribution of bottom decay to the non-photonic electron yields as a function of $p_{T}\left(2.0<p_{T}<9.0 \mathrm{GeV} / c\right)$. For technical details of this analysis, please refer to Ref. [8, 9].

The other one is by measuring the azimuthal angular correlations between non-photonic electrons and $D^{0}$ in $p+p$ collisions [10]. MC simulations (PYTHIA and MC@NLO) predict the $e^{-}-D^{0}$ correlation for electrons from $D$ meson decays will dominantly distribute in the away side, while that for electrons from $B$ meson decays will dominantly distribute in the near side (there is still a small amount of away side contribution). In the Barrel EMC tower triggered sample, $D^{0}$ reconstructed through $K^{-} \pi^{+}$channel is significantly enhanced compared to the minimum bias data sample. The signal-to-background ratio was improved by a factor of $\sim 100$ compared to that obtained in $d+$ Au minimum bias collisions. The azimuthal correlation function of $e^{-}$ $D^{0}$ (and $e^{+}-\overline{D^{0}}$ ) was fit to the sum of two components, and one can extract the relative bottom contributions to the electron yields.

The relative contribution of electrons from $B$ meson decays to the non-photonic electron yields $\left(R_{B}\right)$ estimated by the two approaches is shown in Fig. 2.5, compared with the FONLL predictions. We can see $R_{B}$ increases with $p_{T}$ and it becomes comparable to the contribution from $D$ meson decay around $5.0 \mathrm{GeV} / c$. Our result is consistent with the FONLL calculation within the uncertainties.

In the $e-D^{0}$ analysis, MC calculations show the near side correlation is essentially from $B$ meson decays, and the gluon splitting process for $D$ production is negligible. The multiplicity of heavy quark pairs in gluon jets is calculable in $\mathrm{pQCD}$, and the leading nonperturbative correction is believed to be small [11]. Thus, experimentally measuring the charm yields from gluon jet splitting becomes important. 


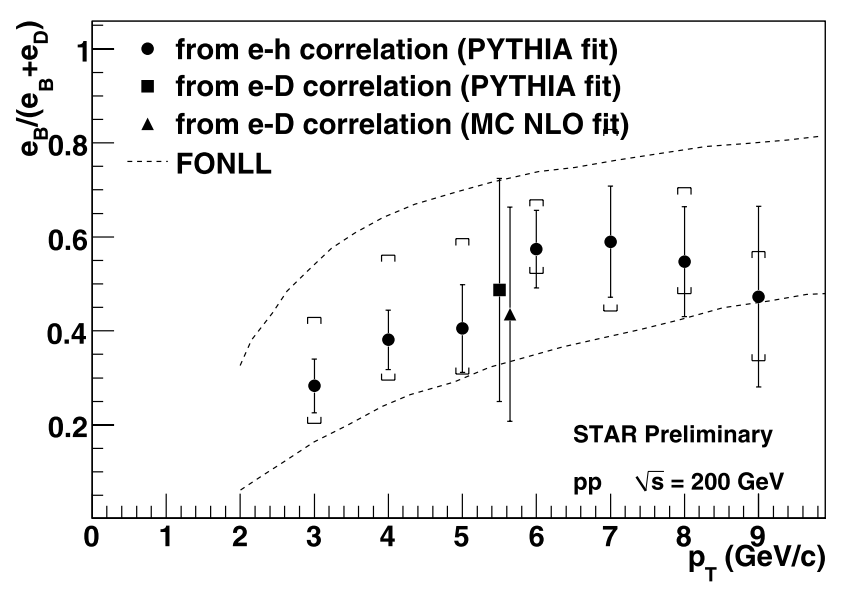

Fig. 2.5 Transverse momentum dependence for the relative contribution of electron from $B$ meson to the non-photonic electron yields from $e-h$ and $e-D^{0}$ azimuthal angular correlation analysis. The curves are FONLL calculations

The measurement is done by measuring the $D^{* \pm}$ yields in the reconstructed jets. $D^{* \pm}$ was reconstructed through the standard method of searching for the decay sequence $D^{*+} \rightarrow D^{0} \pi^{+}, D^{0} \rightarrow K^{-} \pi^{+}$(and its charge conjugate channel) by plotting the invariant mass difference $\Delta M=$ $M\left(K^{\mp} \pi^{ \pm} \pi^{ \pm}\right)-M\left(K^{\mp} \pi^{ \pm}\right)$. Jets were reconstructed using a mid-point cone algorithm, and the jet reconstruction is described in Ref. [12]. Then we correlated the $D^{* \pm}$ with the reconstructed jets, and calculated the fragmentation variable $z \equiv p_{L}\left(K^{\mp} \pi^{ \pm} \pi^{ \pm}\right) / E_{\text {jet }}$ for the near side signal candidates, which is shown in Fig. 2.6 upper plot. The uncertainties represented by bars are statistical and the brackets indicate the contribution caused by combinatorial background subtraction. No correction were made in this plot for trigger and reconstruction efficiency. The MC data shown in Fig. 2.6 upper plot were generated using PYTHIA with only direct charm flavor creation processes $(g g \rightarrow c \bar{c}$, and $q \bar{q} \rightarrow c \bar{c})$. The simulated data were analyzed in the same way as the real data and were normalized using the total charm production cross section measured by STAR [3]. The high $z$ data points are well reproduced by the simulation with direct flavor creation processes, while the excess in the low $z$ region is expected to originate from production processes that are not included in the simulation, such as gluon splitting.

After efficiency and acceptance corrections, we obtain $N\left(D^{* \pm}\right) / N($ jet $)=0.015 \pm 0.008 \pm 0.007$ for $0.2<z<0.5$ with mean jet energy $\left\langle E_{T}\right\rangle=11.5 \mathrm{GeV}$. To get the ratio of gluon splitting into charm pairs $R_{g \rightarrow c \bar{c}}$, one needs to correct for the gluon jet fraction, the charm quark to $D^{*+}$ fragmentation fraction, and the $z$ coverage. The final gluon splitting rate data point from STAR, together with measurements from other experiments $[14,15]$ are shown in Fig. 2.6 lower plot. The datum point is consistent with pQCD evaluation
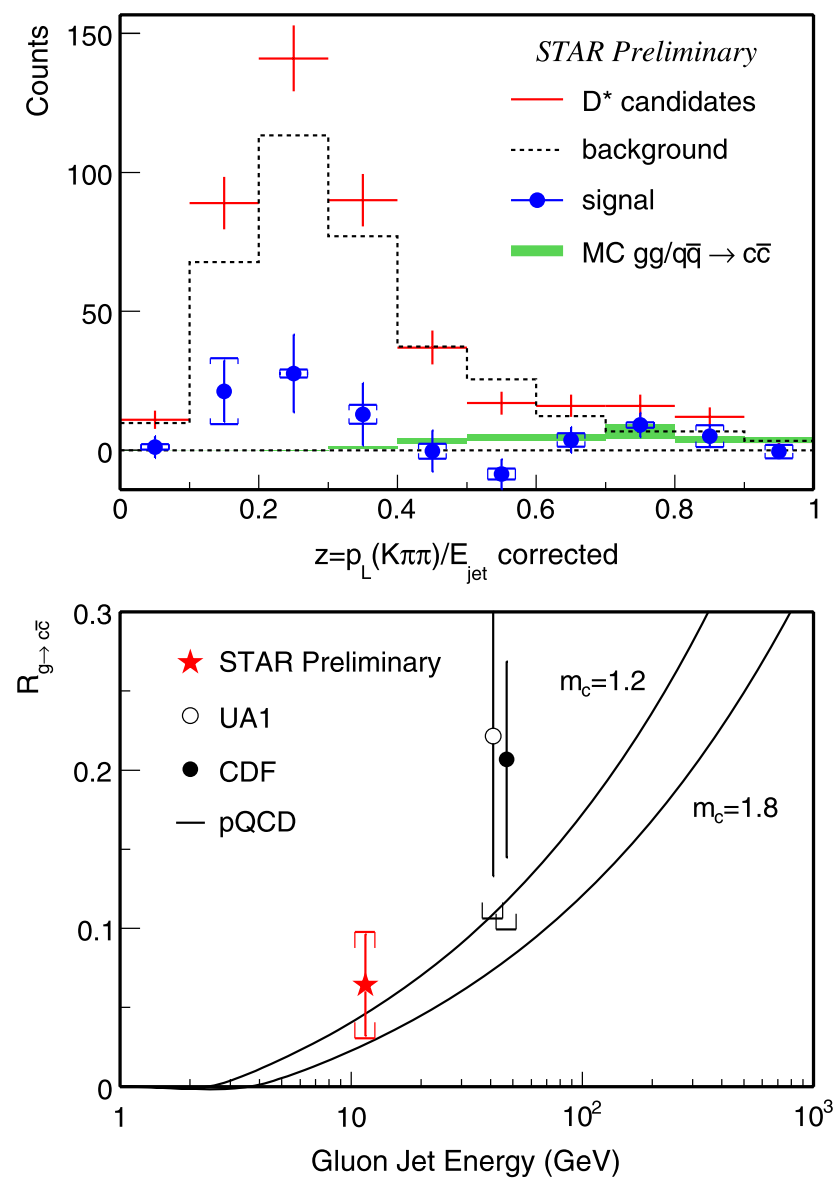

Fig. 2.6 Upper: $D^{*}$ in jet fragmentation variable $z$ distributions from Barrel JP2 data set compared with the full MC simulation with only direct flavor creation process. The excess in low $z$ region is expected from higher order processes. Lower: Gluon splitting rate to charm pairs as a function of the gluon jet energy. Measurements from STAR, UA1, and $\mathrm{CDF}$ collaborations are compared with $\mathrm{pQCD}$ calculations

of gluon splitting into a pair of charm quarks and subsequent hadronization into $D^{* \pm}$ mesons [11]. To gain insight in the production mechanism of charmed hadrons at RHIC, we use the measured jet differential cross section [13] and the gluon splitting rate [11] to estimate the contribution of gluon jet production to the total charm production cross section. We find that for $p_{T}>2 \mathrm{GeV} / c$, charm production in gluon jets is more than one order of magnitude smaller than the total charm cross section $[1,2]$. Hence the charm content in jets at RHIC energies has a small contribution from gluon splitting and is dominated by jets initiated by charm quarks.

\section{Summary and outlook}

The STAR detector is unique to perform multi-approach measurements on heavy quark production in midrapidity at RHIC. STAR has made extensive measurements on open 
charm hadron reconstruction through its hadronic decay channel, non-photonic electrons, heavy quark decay muons and heavy quark triggered correlations. So far, the conclusions achieved still have large uncertainties. To quantify the medium properties and Equation-Of-State, precision measurements on open heavy quark production are called for. Topological reconstruction of identified open heavy quark hadrons is essential in this direction. So the current subdetector upgrade plans in pipe for the STAR detector are very important to this goal. The Heavy Flavor Tracker proposal [16] will allow us to reconstruct the secondary vertices of open charm decays with much lower background. The ongoing Time-Of-Flight project will improve the hadron particle identification capability significantly; hence improve the signal-to-background ratio for charm hadron reconstruction.

\section{References}

1. M. Cacciari et al., Phys. Rev. Lett. 95, 122001 (2005)

2. R. Vogt, arXiv:0709.2531

3. J. Adams et al., Phys. Rev. Lett. 94, 062301 (2005)

4. B.I. Abelev et al., arXiv:0805.0364

5. B.I. Abelev et al., Phys. Rev. Lett. 98, 192301 (2007)

6. A. Shabetai et al., in QM08 Proceedings

7. H. Liu et al., Phys. Lett. B 639, 441 (2006)

8. X. Lin et al. (STAR Collaboration), J. Phys. G 34, S821 (2007)

9. G. Wang et al. (STAR Collaboration), in QM08 Proceedings

10. A. Mischke et al. (STAR Collaboration), These Proceedings

11. A.H. Mueller, P. Nason, Phys. Lett. B 157, 226 (1985)

12. B.I. Abelev et al., Phys. Rev. Lett. 100, 232003 (2008)

13. B.I. Abelev et al., Phys. Rev. Lett. 97, 252001 (2006)

14. C. Albajar et al., Phys. Lett. B 244, 566 (1990)

15. F. Abe et al., Phys. Rev. Lett. 64, 348 (1990)

16. STAR HFT Collaboration, STAR HFT Proposal 